\title{
Assessment of potential short-term effects of intermittent UMTS electromagnetic fields on blood circulation in an exploratory study, using near-infrared imaging
}

\author{
Spichtig, Sonja ; Scholkmann, Felix ; Chin, Lydia ; Lehmann, Hugo ; Wolf, Martin
}

\begin{abstract}
Potential short-term effects of intermittent UMTS electromagnetic fields (UMTS-EMF) on the cerebral blood circulation were assessed by near-infrared imaging. Different UMTS-EMF signals and exposure positions were applied in an exploratory study on eight subjects. Since no significant changes were found, the UMTS-EMF signal and the exposure position with the maximum changes in the measurement variables were estimated.
\end{abstract}

DOI: https://doi.org/10.1007/978-1-4614-1566-4_13

Posted at the Zurich Open Repository and Archive, University of Zurich

ZORA URL: https://doi.org/10.5167/uzh-70588

Book Section

Accepted Version

Originally published at:

Spichtig, Sonja; Scholkmann, Felix; Chin, Lydia; Lehmann, Hugo; Wolf, Martin (2012). Assessment of potential short-term effects of intermittent UMTS electromagnetic fields on blood circulation in an exploratory study, using near-infrared imaging. In: Wolf, Martin; et al. Oxygen Transport to Tissue XXXIII. New York, NY: Springer, 83-88.

DOI: https://doi.org/10.1007/978-1-4614-1566-4_13 


\title{
Assessment of potential short-term effects of intermittent UMTS electromagnetic fields on blood circulation in an exploratory study, using near-infrared imaging
}

\author{
Sonja Spichtig ${ }^{1}$, Felix Scholkmann ${ }^{1}$, Lydia Chin ${ }^{1}$, Hugo Lehmann ${ }^{2}$, Martin Wolf ${ }^{1}$ \\ ${ }^{1}$ University Hospital Zurich, Division of Neonatology, Department of Obstetrics and Gynecology, Biomedical Optics Research Laboratory, 8091 \\ Zurich, Switzerland \\ ${ }^{2}$ Swisscom (Switzerland) Ltd., Innovation Competence Centre, Environment \& Electromagnetic Compatibility, 3050, Bern, Switzerland
}

\section{Introduction}

We investigated potential short-term effects (occurring within $80 \mathrm{~s}$ ) of intermittent Universal Mobile Telecommunications System electromagnetic fields (UMTS-EMF) on blood circulation in the human head using near-infrared imaging (NIRI). NIRI measures changes in oxy, deoxy, and total hemoglobin concentration $\left(\Delta\left[\mathrm{O}_{2} \mathrm{Hb}\right], \Delta[\mathrm{HHb}]\right.$, and $\Delta[\mathrm{tHb}]$ ), which reflect changes in cerebral activity, perfusion, and/or oxygenation. Its insensitivity to EMF, the high temporal resolution, and the flexibility in choosing the exposure sequence makes NIRI an excellent tool to study immediate physiological effects of continuous and intermittent EMF.

The aim of this exploratory study was (1) to investigate several UMTS signal types and exposure positions in eight subjects for potential effects, and (2) to define a final protocol for a more extensive study.

\section{Materials and Methods}

\subsection{Optical Setup}

Hardware and software changes on the commercially available ISS Oxiplex ${ }^{\circledR}$ (ISS Inc., Champaign, Illinois, USA) quadrupled the number of detector and light source combinations available for a single optical sensor. The available 2 detectors and 8 source positions enable to collect in total 16 light bundles , also called light paths. Each light bundle comprised light at two wavelengths, namely 690 and $830 \mathrm{~nm}$. The NIRI sensor was custom built in our lab and contains no active parts to be insensitive to UMTS-EMF. Ten-meter long optical fibers were used for the NIRI sensor to enable the placement of the NIRI instrument outside of the exposure chamber and therefore to minimize potential electrical interferences. For more details about the modifications on the ISS Oxiplex ${ }^{\circledR}$ refer to [1].

\subsection{Exposure Setup}

The measurements were carried out in an experimental room located in the basement of the University Hospital Zurich, where the electromagnetic background fields were determined to be very low $@ 1.9 \mathrm{GHz}$ the background electrical field strength was $0.0002 \mathrm{~V} / \mathrm{m}$ ). The noticeable contributions to the background electrical field strength were a GSM downlink signal (@900 MHz: $0.005 \mathrm{~V} / \mathrm{m}$ ), a FM radio signal (@88 MHz: $0.0005 \mathrm{~V} / \mathrm{m}$ ), and the hospital pager (@451 MHz: 0.0003 V/m).

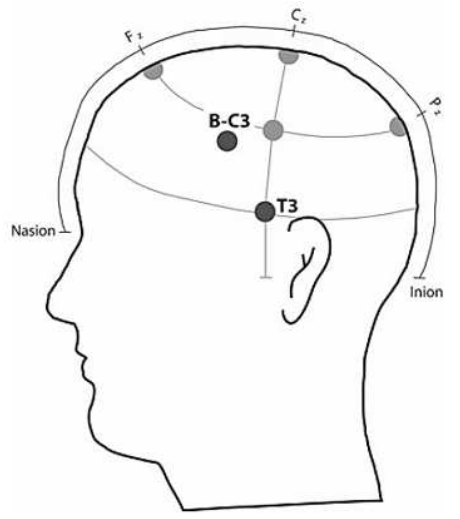

Fig. 13.1: Graphical representation of the exposure positions T3 and B-C3 in the international 10-20 EEG positioning system [11].

Absorber walls were arranged around the subject's bench to minimize possible UMTS-EMF reflections of the exposure setup.

The electronics was programmed to produce four different UMTS signal types with a carrier frequency of $1.9 \mathrm{GHz}$ (1) continuous wave (cw), (2) base station-like (bs, as in the TNO-study [2]), (3) W-CDMA uplink 1 communication channel (1up; similar to the signal proposed by Ndoumbe Mbonjo et al. [3], (4) W-CDMA uplink 6 communication channels (6up; as proposed by Ndoumbe Mbonjo et al. [3]). Two exposure positions were defined, one located close to the ear (Fig. 13.1: T3) and the other (Fig. 13.1: B-C3) midway between the motor cortex and position $\mathrm{B}$ where previously a change in the regional cerebral blood flow was reported for a Global System for Mobile Communications (GSM) signal $[4,5]$. The center point of the optical sensor was positioned directly on these positions and a planar patch antenna (SPA 2000/80/8/0/V, Huber \& Suhner, Switzerland) emitting the EMF at a distance of $4 \mathrm{~cm}$.

The spatial peak specific absorption rate (SAR) over $10 \mathrm{~g}$ of the exposure setup was simulated with SEMCAD $\mathrm{X}$ (Version 13, Schmid and Partner Engineering, Zurich, Switzerland) using the Specific Anthropomorphic Mannequin (SAM) phantom defined in CENELEC 50361 [6]. The simulation results were verified with an experimental SAR measurement using the DASY5 Dosimetric Assessment System (Schmid and Partner Engineering, Zurich, Switzerland) at the IT'IS foundation in Zurich. The simulated and experimental data showed deviations smaller than $\pm 5 \%$ and thus a high agreement. Maximum peak SAR (over $10 \mathrm{~g}$ ) applied was $1.8 \mathrm{~W} / \mathrm{kg}$, which is below the 
ICNIRP guidelines. For more information about the exposure system and dosimetry see [7].

\subsection{Subjects and Study Protocol}

Eight right-handed male subjects (age: $28.6 \pm 2.9$ years) participated after giving written informed consent. They were asked to abstain from coffee, cigarettes, and mobile phone use within $2 \mathrm{~h}$ before a measurement. Each subject underwent eight measurements on different days experiencing all possible combinations of UMTS signal types and exposure positions in a single-blind paradigm.

A measurement consisted of 15 cycles, the exposure segments, $20 \mathrm{~s}$ of UMTS-EMF exposure alternated with 60 $\mathrm{s}$ recovery. Before and after the cycles a baseline was recorded for $3 \mathrm{~min}$. In total, a measurement lasted $26 \mathrm{~min}$.

\subsection{Data Analysis}

Data were band pass filtered $(0.008-0.8 \mathrm{~Hz})$ to remove high frequency noise and very low physiological contributions. $\Delta\left[\mathrm{O}_{2} \mathrm{Hb}\right]$ and $\Delta[\mathrm{HHb}]$ were calculated using the modified Lambert-Beer law. The differential pathlength factors (DPF) for C3 given in the literature [8] were linearly interpolated to $\mathrm{DPF}_{690 \mathrm{~nm}}=8.21$ and $\mathrm{DPF}_{830 \mathrm{~nm}}=7.3$ and used in the modified Lambert-Beer law.

To reduce present movement artifacts, the algorithm presented by [9] was applied to the concentration time series of $\Delta\left[\mathrm{O}_{2} \mathrm{Hb}\right]$ and $\Delta[\mathrm{HHb}]$. The sum of the MA reduced $\Delta\left[\mathrm{O}_{2} \mathrm{Hb}\right]$ and $\Delta[\mathrm{HHb}]$ yielded $\Delta[\mathrm{tHb}]$.

The last $10 \mathrm{~s}$ of UMTS-EMF exposure $(\mathrm{ON})$ and recovery (OFF) were averaged. The difference between $\mathrm{ON}-\mathrm{OFF}$ was modeled using a linear mixed-effects model with the factors signal type (levels: cw, bs, 1up and 6up), exposure position (levels: T3, B-C3), light path (defined as the different source-detector combinations; levels: light path 1 to light path 16), and exposure segments (levels: exposure segment 1 to exposure segment 15). The subject was defined as random factor.
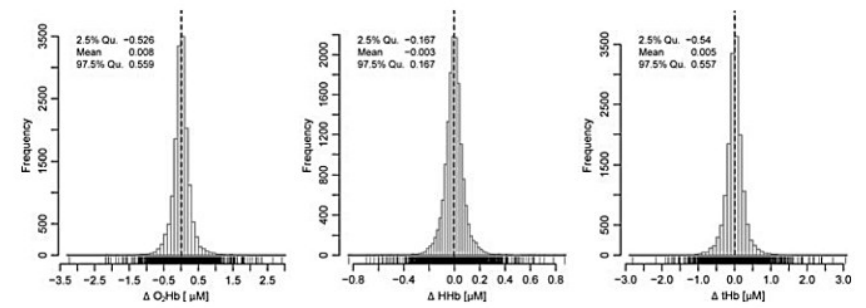

Fig. 13.2: Histograms of $\Delta\left[\mathrm{O}_{2} \mathrm{Hb}\right], \Delta[\mathrm{HHb}]$, and $\Delta[\mathrm{tHb}]$ for all observations. Mean (black dotted vertical line), $2.5 \%$ and 97.5 quantiles are given for each distribution.

\section{Results}

The histograms for the difference between $\mathrm{ON}$ and $\mathrm{OFF}$ are displayed in Fig. 13.2 for $\Delta\left[\mathrm{O}_{2} \mathrm{Hb}\right], \Delta[\mathrm{HHb}]$, and $\Delta[\mathrm{tHb}]$ with all 15'360 observations. They show normally distributed concentration differences with a mean close to zero. No statistically significant differences between exposure and recovery were found for any condition and measurement parameter.

The signal type and exposure position with maximum and minimum differences (ON-OFF) in $\Delta\left[\mathrm{O}_{2} \mathrm{Hb}\right], \Delta[\mathrm{HHb}]$, and $\Delta[\mathrm{tHb}]$ are summarized in Table 13.1.

\section{Discussion and Conclusion}

The largest differences in $\Delta\left[\mathrm{O}_{2} \mathrm{Hb}\right], \Delta[\mathrm{HHb}]$, and $\Delta[\mathrm{tHb}]$ (Table 13.1) occurred for the majority of cases at the posi- tion T3 and the signal type bs. Hence, this signal type and exposure position were selected for the protocol of a more extensive study.

Table 13.1 Maximum and minimum concentration changes in $\mu \mathrm{M}$ described by a linear mixed-effects model

\begin{tabular}{lllr}
\hline $\operatorname{Parameter}$ & Position & Signal & \multicolumn{1}{c}{$[\mu \mathrm{M}]$} \\
\hline $\operatorname{Max}\left(\Delta\left[\mathrm{O}_{2} \mathrm{Hb}\right]\right)$ & $\mathrm{C} 3$ & lup & 0.1357 \\
$\operatorname{Min}\left(\Delta\left[\mathrm{O}_{2} \mathrm{Hb}\right]\right)$ & $\mathrm{T} 3$ & bs & -0.1818 \\
$\operatorname{Max}(\Delta[\mathrm{HHb}])$ & $\mathrm{C} 3$ & cw & 0.0418 \\
$\operatorname{Min}(\Delta[\mathrm{HHb}])$ & $\mathrm{T} 3$ & cw & -0.0380 \\
$\operatorname{Max}(\Delta[\mathrm{tHb}])$ & $\mathrm{T} 3$ & bs & 0.1421 \\
$\operatorname{Min}(\Delta[\mathrm{tHb}])$ & $\mathrm{T} 3$ & bs & -0.1752 \\
\hline
\end{tabular}

To set the size of the obtained changes in perspective, we compared them to concentration changes elicited by functional activation of the brain, e.g., for a motor cortex activation their size is approximately $0.8 \mu \mathrm{M}$ for $\Delta\left[\mathrm{O}_{2} \mathrm{Hb}\right], 0.15$ $\mu \mathrm{M}$ for [HHb], and $0.65 \mu \mathrm{M}$ for $\mathrm{tHb}$. These values are reference values based on the literature including the results of functional measurements on adults in several studies [10]. The changes presented in Table 13.1 were $4-6$ times smaller.

In previous studies $[4,5]$ significant changes in cerebral blood flow (CBF) were detected using PET. Subjects were exposed for $30 \mathrm{~min}$ to a $0.9 \mathrm{GHz}$ handset-like signal mimicking a GSM mobile phone with $1.0 \mathrm{~W} / \mathrm{kg}$ SAR in 16 subjects (for comparison in our study: totally 5 min exposure, $1.9 \mathrm{GHz}$ UMTS-signal, intermittent, $1.8 \mathrm{~W} / \mathrm{kg}$, $8 \mathrm{sub}$ jects). Changes in CBF were measured 10-30 min after exposure, while we analyzed short-term changes during exposure. Significantly increased $\mathrm{CBF}$ was found close to the $\mathrm{C} 3$ position and $<2 \mathrm{~cm}$ deep $[4,5]$, where we measured as well. Any of the discrepant factors may be responsible for not finding significant changes in contrast to the PET studies $[4,5]$.

There could be different reasons why no significances were detected: firstly, the effect could be very small as the detected maximum and minimum differences indicate and the number of subjects was too low to detect the effect with statistical measures. Thus, the power of the study was not sufficient to detect such small effects. And secondly, it could be that there is no effect of UMTS-EMF on the blood circulation at this measurement positions or anywhere.

To further investigate this, a more extensive study with enhanced power (twice the number of subjects) and the exposure parameters mentioned above will be carried out.

Acknowledgments: The authors gratefully acknowledge the support of the Swiss National Foundation (National Research Programme NRP 57). We thank Massimo Merlini (Statistician, ETH Zurich) for his valuable comments and great support.

\section{References}

[1] Spichtig S (2010) Multi-distance and multi-frequency frequency-domain near-infrared spectroscopy: characterization and application. PhD thesis No 19006, Swiss Federal Institute of Technology Zurich

[2] Zwamborn APM, Vossen SHJA, Van Leersum BJAM et al (2003) Effects of global communication system radiofrequency fields on well being and cognitive functions of human subjects with and without subjective complaints. TNO Physics and Electronics Laboratory, FEL-03-C148 
[3] Ndoumbe Mbonjo H, Streckert J, Bitz A et al (2004) Generic UMTS test signal for RF bioelectromagnetic studies. Bioelectromagnetics 25:415-425

[4] Huber R, Treyer V, Borbély AA et al (2002) Electromagnetic fields, such as those from mobile phones, alter regional cerebral blood flow and sleep and waking EEG. J Sleep Res 11(4):289-295

[5] Huber R, Treyer V, Schuderer J et al (2005) Exposure to pulse-modulated radio frequency electromagnetic fields affects regional cerebral blood flow. Eur J Neurosci 21:10001006

[6] CENELEC (2001) Basic standard for the measurement of specific absorption rate related to human exposure to electromagnetic fields from mobile phones (300 MHz-3 GHz). Technical report, EN5036.1

[7] Lehmann H, Pollara L, Spichtig S, Kühn S, Wolf M. Head exposure system for a human provocation study to assess the possible influence of UMTS-like electromagnetic fields on cerebral blood circulation using near-infrared imaging. Bioelectromagnetics. 2011 Aug 12. doi: 10.1002/bem.20688. [Epub ahead of print]

[8] Zhao H, Tanikawa Y, Gao F et al (2002) Maps of optical differential pathlength factor of human adult forehead, somatosensory motor and occipital regions at multiwavelengths in NIR. Phys Med Biol 47:2075-2093

[9] Scholkmann F, Spichtig S, Mühlemann T et al (2010) How to detect and reduce movement artifacts in near-infrared imaging using moving standard deviation and spline interpolation. Physiol Meas 31:649-662

[10] Wolf M, Haensse D, Morren G et al (2006) Do GSM $900 \mathrm{MHz}$ signals affect cerebral blood circulation? A near-infrared spectrophotometry study. Opt Expres 14(13): 6128-41

[11] Jasper HH (1958) Report of the committee on methods of clinical examination in electroencephalography. Electroenceph Clin Neurophysiol 10:370-375 